\section{Revisando conceitos: o papel da psiquiatria moderna no hospital geral e na atenção primária}

\author{
Reviewing concepts: modern psychiatry \\ role in general hospital and at primary care
}

\author{
Sr. Editor, \\ George Engel formulou o modelo biopsicossocial, enfatizando
} a importância de sua aplicação na Medicina como contribuição para a consolidação de um novo paradigma. ${ }^{1}$ As repercussões deste modelo têm se ampliado progressivamente e hoje podemos verificar que a inclusão de aspectos culturais, psicológicos, comportamentais e sociais, ao lado dos biológicos, tornou-se uma diretriz ética na educação médica. ${ }^{2}$ Alimentada pela perspectiva biopsicossocial, a psiquiatria passou a cuidar não apenas dos transtornos mentais dos pacientes, mas também a observar e atuar na dinâmica existente entre equipe de saúde, paciente e seus familiares, contribuindo para o equacionamento dos transtornos relacionais. Passou a participar na construção de um modelo de Medicina Integrada, cuja função é promover a integralidade e a integração dos cuidados. Por integralidade compreende-se a inclusão de diferentes facetas da pessoa e do processo do adoecer; por integração, a articulação de diferentes formas assistenciais do sistema de saúde no âmbito da complexidade dos cuidados. ${ }^{2}$ Objetiva-se evitar a fragmentação da assistência, resultante da necessidade de uma crescente especialização, priorizando a construção de uma especialização integrada (focalizada num campo de conhecimento sem perder a articulação com as demais dimensões do ser e do cuidado). ${ }^{3}$ É frente a este cenário que as atribuições do psiquiatra o colocam, de um lado, como mais um especialista a ocupar-se de sua área específica e, de outro, como um dos articuladores privilegiados do processo de integração dos cuidados. Esta é a tendência atual de uma Psiquiatria moderna, inserida no hospital geral e na atenção primária, que vem sendo discutida e proposta pela Academy of Psychosomatic Medicine, nos Estados Unidos, pela European Consultation-Liaison Workgroup, na Europa, e pelo Departamento de Interconsulta da Associação Brasileira de Psiquiatria, entre outros.

A Psiquiatria se dedica ao estudo das perturbações do contato com a realidade, envolvendo um compromisso com uma epistemologia da observação e com o treinamento dos demais profissionais de saúde para o reconhecimento destas mesmas alterações, seja na forma de transtornos da vida mental do paciente quanto na forma de distúrbios da relação e do campo assistencial. Neste processo de modernização de suas ações, destacam-se as funções a serem desenvolvidas pelo psiquiatra no hospital geral e na atenção primária no sentido de assessorar e contribuir com as equipes para a promoção das seguintes metas:

1) Deslocamento do foco de atenção, da doença como evento para o adoecer como processo;

2) Continuidade dos cuidados; ${ }^{4}$

3) Otimização da abrangência dos cenários de atendimento; 5

4) Adequação dos programas de ensino e promoção de cuidados de saúde para os estudantes e profissionais;

5) Educação permanente de pacientes e familiares;
6) Promoção do trabalho multiprofissional.

A contribuição dessa Psiquiatria moderna no hospital geral e na atenção primária necessita ser continuamente aprimorada. Para tanto, é importante garantir a formação destes profissionais que, em sua atividade, estejam capacitados a incorporar tanto a sua intervenção como especialistas em transtornos mentais, quanto a sua intervenção no processo de assessorar e catalisar a promoção de ações que contemplem a integralidade e a integração das práticas.

Mario Alfredo De Marco, Vanessa de Albuquerque Cítero, Luiz Antonio Nogueira Martins Departamento de Psiquiatria, Universidade Federal de São Paulo (UNIFESP), São Paulo (SP), Brasil

Finaciamento: Inexistente

Conflito de interesses: Inexistente

\section{Referências}

1. Engel GL. The need for a new medical model: a challenge for biomedicine. Science. 1977;196(4286):129-36.

2. Smith G, Clarke D. Assessing the effectiveness of integrated interventions: terminology and approach. Med Clin N Am. 2006;90(4):533-48.

3. De Marco MA, organizador. A face humana da Medicina: do modelo biomédico ao modelo biopsicossocial. São Paulo: Casa do Psicólogo; 2003. p. 38-41.

4. Hjortdahl P, Laerum E. Continuity of care in general practice: effect on patient satisfaction. BMJ. 1992;304(6837):1287-90.

5. Pelikan J, Krajic K, Dietscher C. The health promoting hospital: concept and development. Patient Educ Couns. 2001;45(4):239-43.

\section{Marcadores antropométricos de sobrepeso e Inventário Beck de Depressão: uma associação a ser considerada}

\author{
Anthropometric indicators of overweight \\ and the Beck Depression Inventory: an \\ association to be considered
}

\section{Sr. Editor,}

Gostaríamos de comentar sobre alguns resultados que chamaram nossa atenção em um dos trabalhos desenvolvidos por nosso grupo. A obesidade (Índice de Massa Corporal [IMC] > $30 \mathrm{~kg} / \mathrm{m}^{2}$ ), e principalmente o excesso de peso (IMC > $25 \mathrm{e}$ $<30 \mathrm{~kg} / \mathrm{m}^{2}$ ), já são hoje considerados epidemias mundiais, acarretando uma elevada morbi-mortalidade. ${ }^{1}$ Além de todo 0 impacto clínico relacionado ao sobrepeso, existe também um aumento de diversas comorbidades psiquiátricas. 
Recentemente, nosso grupo demonstrou que existe uma relação direta entre marcadores antropométricos de sobrepeso (IMC e cintura abdominal) e os sintomas depressivos. ${ }^{2}$ A avaliação dos sintomas depressivos, entretanto, levantou algumas dúvidas interessantes. O Inventário Beck de Depressão (IBD), instrumento utilizado em nosso estudo, avalia tanto sintomas cognitivos como somáticos da depressão. Especificamente, diversos dos itens somáticos (por exemplo, fadiga, libido, dificuldades para o trabalho) podem estar muito mais relacionados ao excesso de peso do que a própria depressão, levando a resultados falso-positivos.

Desta forma, complementamos nossa análise de modo a melhor avaliar a relação de peso corporal com sintomas depressivos. Foram avaliados 221 pacientes obesos $\left(\mathrm{IMC}=39,9 \pm 6,8 \mathrm{Kg} / \mathrm{m}^{2}\right)$, entre 18 e 77 anos $(38,8 \pm 11,8)$, que procuraram tratamento para emagrecer no Grupo de Obesidade e Transtornos Alimentares (GOTA) do Instituto Estadual de Diabetes e Endocrinologia (IEDE). O protocolo foi aprovado pelo Comitê de Ética em Pesquisa da Instituição. Os dados antropométricos foram obtidos por um endocrinologista e todos os pacientes preencheram a versão em português do IBD. ${ }^{3}$ Os dados demográficos incluíram renda familiar, estado civil, grupo étnico e escolaridade.

A gravidade dos sintomas depressivos foi correlacionada com aspectos demográficos e antropométricos. Correlações não significativas foram encontradas entre o IBD e o estado civil $(r=0,04 ; p=0,50)$, idade $(r=0,01 ; p=0,79)$ e grupo étnico $(r=0,03 ; p=0,56)$. Por outro lado, uma correlação significativa foi demonstrada entre o IBD e o IMC $(r=0,17$; $p=0,011)$, renda familiar $(r=-0,20 ; p=0,0047)$ e nível educacional ( $r=-0,13 ; p=0,047)$. Após regressão linear multivariada, a única variável que continuou mostrando uma correlação quase significativa com o IBD foi o IMC ( $p=0,054)$.

Nossos resultados levam a algumas considerações importantes. Parece existir, pelo menos em pacientes obesos, uma relação direta entre o sobrepeso e o $I B D$, independente de variáveis sociodemográficas. Estes achados podem sugerir que o aumentodo IMC se relaciona a um aumento da depressão ou, por outro lado, que existe uma interferência do peso na avaliação dos sintomas depressivos pelo IBD. Por exemplo, em dois artigos publicados recentemente nesta revista, os autores utilizam o IBD para avaliação dos sintomas depressivos. ${ }^{4-5}$ Embora os autores avaliem diversas variáveis sociodemográficas e psicopatológicas, em nenhum momento os dados antropométricos foram levados em conta.

O IBD, devido a sua fácil aplicação e boa confiabilidade, é largamente utilizado na psiquiatria. A maioria dos estudos leva em conta diversas variáveis que podem interferir com o IBD, de modo a corrigir os diversos fatores de confusão existentes. As variáveis antropométricas, entretanto, são, na maioria das vezes, esquecidas. Nossa sugestão aos autores desta revista é que, devido à elevada prevalência de pacientes com obesidade e sobrepeso na população geral (e provavelmente em suas amostras), que sejam incluídos nas análises os marcadores de obesidade, de modo a excluir mais um importante fator de confusão.

Rodrigo 0 Moreira Grupo de Obesidade e Transtornos Alimentares (GOTA), Instituto Estadual de Diabetes e Endocrinologia (IEDE) / Instituto de Psiquiatria da Universidade Federal do Rio de Janeiro (IPUB/UFRJ), Rio de Janeiro (RJ), Brasil Escola Médica de Pós-Graduação, Pontifícia Universidade Católica do Rio de Janeiro (PUC-RJ), Rio de Janeiro (RJ), Brasil
Mário S Zen

Instituto Estadual de Diabetes e Endocrinologia do Rio de Janeiro (IEDE), Rio de Janeiro (RJ), Brasil

Financiamento: Inexistente

Conflito de Interesse: Inexistente

Referências

1. Flegal KM, Carroll MD, Ogden CL, Johnson CL. Prevalence and trends in obesity among US adults, 1999-2000. JAMA. 2002;288(14):1723-7.

2. Moreira RO, Marca KF, Appolinário JC, Coutinho WF. Increased Waist Circumference is associated with an increased prevalence of mood disorders and depressive symptoms in obese women. Eat Weight Disord. 2007, In press.

3. Gorenstein C, Andrade LHSG, Zuardi AW. Escalas de avaliação clínica em psiquiatria e psicofarmacologia. Rev Psiquiatr. Clin. 1998;26:65-9.

4. Cigognini MA, Furlanetto LM. Diagnóstico e tratamento dos transtornos depressivos em um hospital geral. Rev Bras Psiquiatr. 2006;28(2):97-103.

5. Mello VA, Malbergier A. Depressão em mulheres infectadas pelo HIV. Rev Bras Psiquiatr. 2006;28(1):10-7.

\section{Treatment of comorbid attention deficit hyperactivity disorder and depression in pediatric patient} Tratamento do transtorno de déficit de atenção com hiperatividade e depressão coexistentes em paciente pediátrico

\section{Dear Editor,}

The comorbidity between attention deficit hyperactivity disorder (ADHD) and mood disorders has been a controversial issue. ${ }^{1}$ There are evidence that mood disorders and ADHD may be comorbid disorders, that ADHD and bipolar disorder may be related, and that depression may be an outcome of ADHD. ${ }^{1}$ In the case presented below, comorbid ADHD and depression were successfully treated with a combination of bupropion and cognitive behavioral therapy.

P., 7 years old, male, had been diagnosed with ADHD when he was 2 years old. There was no family history of psychopathology. Different health professionals had treated him with several medications without improvement. P. was referred by a neurologist because he was having severe behavioral problems. He was violent, defiant, and irritable, had low selfesteem and struggled to express himself in an appropriate manner. He had not learned to read and write, and his drawings were bizarre. His parents reported that P. experienced anxiety, sadness, fear, frequent crying, tantrums, self-mutilation and ideas of death. At the interview, P. was restless, avoided eye contact, presented blunted affect, and dysphoric mood. His speech was disorganized and incoherent. His walking was spastic and lethargic. P. was medicated with regular 